\title{
Loss of Estrogen Receptor Alpha (ER $\alpha$ ) Exacerbates Experimental Pulmonary Arterial Hypertension (PAH)
}

\author{
Annie Gensel (M.S.) ${ }^{1}$, Andrea Frump (Ph.D.) ${ }^{2}$, Tim Lahm (M.D.) $)^{2,3}$ \\ ${ }^{1}$ Indiana University School of Medicine, ${ }^{2}$ Indiana University School of Medicine, Department of \\ Medicine, Division of Pulmonary, Critical Care, Sleep and Occupational Medicine, ${ }^{3}$ Roudebush \\ VA Medical Center
}

Background and Hypothesis: PAH is a sexually dimorphic cardiopulmonary disease characterized by excessive vasoconstriction and pulmonary artery remodeling, leading to right ventricular (RV) failure and death. While women are more likely to develop PAH, they exhibit more favorable hemodynamics and increased survival compared to men. These improved outcomes in women with PAH have been linked to protective effects of the sex steroid 17ßestradiol (E2). While E2's receptor ERa is protective in the systemic vasculature, its function in the cardiopulmonary system has not been explored. We hypothesized that loss of ERa exacerbates $\mathrm{PAH}$.

Experimental Design: Studies were performed in male and female wild type (WT) or ERa lossof-function mutant ( $\mathrm{ERa}^{\mathrm{mut}}$ ) rats with monocrotaline (MCT)-induced PAH as well as disease-free controls. We quantified hemodynamics (RV catheterization), RV structure and function (echocardiography) and pulmonary artery remodeling (Verhoff-van Giesson staining). Lung tissues were analyzed for expression of pulmonary vascular homeostatic regulators BMPR2 and apelin and pro-survival regulator ERK (Western blot). $\mathrm{P}<0.05$ (ANOVA) was considered significant.

Results: $\mathrm{ERa}^{\text {mut }}$ rats did not differ hemodynamically from WT controls. However, after MCT administration, ERa ${ }^{\text {mut }}$ rats exhibited more severe disease than WT MCT rats (demonstrated by increased RV hypertrophy, RV systolic pressure, total pulmonary resistance index, as well as decreased cardiac index and stroke volume index $(p<0.05)$. Interestingly, female ERa ${ }^{\text {mut }} M C T$ rats exhibited more severe disease than their male counterparts. Apelin expression decreased in $\mathrm{ERa}^{\text {mut }} \mathrm{MCT}$ lungs compared to WT and $\mathrm{ERa}^{\text {mut }}$ controls $(\mathrm{p}<0.05)$. Furthermore, female WT MCT lungs exhibited preserved apelin expression compared to male WT MCT $(p<0.05)$. BMPR2 expression in ERa ${ }^{\text {mut }}$ MCT lungs decreased compared to WT and ERa ${ }^{\text {mut }}$ controls, as well as WT MCT $(p<0.05)$.

Conclusion: Loss of ERa aggravates MCT-PAH, indicating that ERa exerts protective effects in the cardiopulmonary system. Harnessing ERa signaling may represent a novel treatment strategy for women and men with $\mathrm{PAH}$. 